\title{
Competencias matemáticas de ingenieros informáticos egresados: un estudio descriptivo
}

\author{
Juan Jesús Barbarán Sánchez. Universidad de Granada \\ José Antonio Fernández Bravo. Universidad Complutense de Madrid \\ Javier Barbarán Sánchez. Universidad de Málaga
} Recepción: 23 de junio de 2016 | Revisión: 30 de junio de 2016 | Aceptado: 11 julio de 2016
Correspondencia: barbaran@ugr.es
Citar: Barbaran, JJ., Fernandez, JA. y Barbaran, J. (2016). Competencias matemáticas de ingenieros informáticos egresados: un
estudio descriptivo. ReiDoCrea, 5, 146-160.

Resumen: El enfoque competencial de la enseñanza es una de las principales novedades que incluye el Espacio
Europeo de la Educación Superior (EEES). De acuerdo con esta perspectiva, resulta interesante determinar qué
competencias ponen en práctica los egresados de cualquier titulación en su trabajo. En este artículo presentamos los
resultados de una investigación descriptiva en la que analizamos las competencias matemáticas que usan los
ingenieros informáticos en el ejercicio de su profesión. Para ello, se seleccionó una muestra incidental formada por 414
sujetos egresados de diferentes universidades españolas. La metodología utilizada es cuantitativa y ha constado de las
siguientes fases: diseño de la encuesta, validación de la misma a través de una prueba piloto y por parte de expertos,
distribución de la encuesta entre los egresados y análisis de los resultados. Los datos analizados muestran que los
ingenieros informáticos egresados utilizan las competencias matemáticas en su labor profesional siendo la
competencia algebraica la más usada y la competencia numérica la que consideran que necesitarían más formación
académica que la recibida en sus estudios universitarios para desempeñar de manera eficiente su labor profesional.
Este hecho nos revela la importancia que tiene el desarrollo de la citada competencia en su formación académica.

Palabras clave: Competencias | Matemáticas

Mathematical competencies of graduate computer engineers: a descriptive study

Abstract: The competence approach of teaching is one of the main new features included in the European Higher Education Area (EHEA). According to this perspective, it is interesting to determine what competencies implement any degree graduates in their work. This paper presents the results of a descriptive research that analyzed the math competencies used by computer engineers in their profession. To do this, an incidental sample of 414 graduate subjects from different Spanish universities was selected. The methodology is quantitative and has consisted of the following phases: survey design, validation thereof through a pilot test and by experts, survey distribution among graduates and analysis of results. The data analyzed show that graduate computer engineers use mathematical competencies in their professional job and algebraic competence is the most used and numerical competence the one they need more education than received in university to perform their professional job efficiently. This reveals the importance of the development of that competence in their education.

Keywords: Competences | Mathematics

\section{Introducción}

\section{Planteamiento del problema y delimitación de los objetivos de la investigación}

En la sociedad actual una de las profesiones más demandadas es la de Ingeniero Informático. Estos profesionales deben estar preparados para trabajar en empresas que están en un continuo proceso de innovación. La formación que reciben estos profesionales tiene una importante base matemática como se refleja en su plan de estudios, basado en el Libro Blanco del Título de Grado en Ingeniería Informática elaborado por ANECA (Agencia Nacional de Evaluación de la Calidad y Acreditación) (ANECA, 2004). Estos títulos se imparten actualmente en aproximadamente 50 universidades españolas (públicas y privadas) dentro del Espacio Europeo de Educación Superior (EEES). Este espacio europeo enfatiza la incorporación de modelos de formación orientados al dominio de las competencias; la introducción de este nuevo enfoque puede concebirse como una tentativa interesante y sistemática para poner la formación universitaria a la altura de los nuevos tiempos (López Ruiz, 2010). Las competencias son de utilidad en numerosos ámbitos de su actividad profesional a largo plazo (Radcliffe, 2006). 
Dos de las preguntas formuladas en el Tertiary Education for the Knowledge Society, realizado por la Organización para la Cooperación y el Desarrollo Económico (OCDE, 2008) y recogidas en López Ruiz (2010) son:

- ¿Reciben los universitarios una educación pertinente o existe un desajuste entre las materias que ellos estudian y las necesidades de la economía?

- ¿Son apropiadas las capacidades y habilidades que los jóvenes adquieren para el desempeño de los puestos exigidos en el mundo laboral?

El egresado de cualquier titulación universitaria debería tener la capacidad de resolver las situaciones problemáticas que se encuentra en su labor profesional. Estudiar la inserción laboral de los egresados de las diferentes titulaciones universitarias es una línea de trabajo relevante (Ávila \& Aguirre, 2005; Freire, Teijeiro \& Pais, 2013; Vidal, 2003; Jaramillo, Giraldo \& Ortiz, 2006; Tascón, Álvarez, Couto, Gutiérrez \& Aguado, 2013). Concretamente, en lo que se refiere a la Ingeniería Informática destacamos el trabajo llevado a cabo por Pérez Iglesias (2012) en el que estudia las competencias que demandan los egresados comparando las opiniones de egresados de España y Portugal, y el realizado por Carbonell (2002) en el que analiza los estudios universitarios en Informática a través de sus egresados en el caso concreto de la Universidad de Alicante en el periodo 1984-2001. Vintere \& Zeidmane (2014) analizan las matemáticas usadas por graduados de diferentes titulaciones en su práctica profesional y las necesidades de mejora en el conocimiento matemático que presentan. Un aspecto que consideramos de especial interés es conocer las competencias que los ingenieros informáticos usan en su trabajo $y$, más concretamente, las competencias matemáticas. Esta investigación describe de forma detallada cuáles son las competencias matemáticas que desarrollan los estudiantes de Ingeniería Informática durante su carrera. Nos hemos planteado los siguientes objetivos:

- Mostrar las competencias matemáticas más usadas por los profesionales egresados de Ingeniería Informática en el ejercicio de su profesión.

- Determinar las competencias matemáticas sobre las que los ingenieros informáticos egresados necesitarían más formación académica que la recibida en sus estudios universitarios para desempeñar de manera eficiente su labor profesional.

Este trabajo viene a complementar estudios anteriores como el llevado a cabo por Nguyen (1998), el informe del consorcio Career Space (2001) titulado "Perfiles de capacidades profesionales genéricas de TIC. Capacidades profesionales futuras para el mundo del mañana" donde se presenta una relación de las competencias específicas en relación con unos perfiles profesionales ya definidos, la "Propuesta de Acciones para la Formación de profesionales en Electrónica, Informática y Telecomunicaciones" promovido por la Fundación Tecnologías de la Información, el Colegio Oficial y la Asociación Española de Ingenieros de Telecomunicación (2003) y "Profesiones y Perfiles en Informática" llevado a cabo por la Asociación de Doctores, Licenciados e Ingenieros en Informática (2004). Esta información puede ser de interés para las universidades a la hora de tomar decisiones en lo que se refiere a modificar las competencias de las asignaturas de matemáticas de la titulación y para conectar la formación recibida en la universidad y la realidad del mundo laboral. Además, damos respuesta a lo planteado por Ávila \& Aguirre (2005) en lo que se refiere al seguimiento de los egresados. 


\section{Antecedentes y fundamentación teórica}

El término competencia aparece en la psicología para extenderse posteriormente al mundo laboral (Urrego-Giraldo \& Giraldo, 2009). Teniendo en cuenta que el sistema educativo debe preparar a los estudiantes para su inserción laboral, la Organización para la Cooperación y el Desarrollo Económico (OCDE, 2005) planteó la necesidad de diseñar un modelo educativo basado en que el educando logre aplicar sus conocimientos para resolver problemas de la vida, haciendo hincapié en la faceta profesional. A nivel universitario, este planteamiento fue recogido por el llamado Espacio Europeo de Educación Superior (EEES) que cambió el modelo de enseñanza existente hasta la fecha y demandó que los planes de estudio desarrollasen las competencias con el fin de: acercar el mundo laboral y la formación, adecuar a los profesionales a los cambios en la tecnología y en las organizaciones, renovar la oferta educativa y las modalidades de adquisición y reconocimiento de cualificaciones (ANECA, 2004). Estas competencias van aparejadas al concepto de perfil profesional de los egresados que realizan un determinado trabajo.

En España, los estudios universitarios de Ingeniería Informática fueron reestructurados por el Real Decreto de 26 de febrero de 1976. Posteriormente, el Real Decreto $1497 / 1987$ de 27 de noviembre estableció un sistema de créditos en el que se fijan unos mínimos y máximos por curso y se ordenan las materias troncales, obligatorias, optativas y de libre configuración. Es en el Boletín Oficial del Estado (BOE) de 20 de noviembre de 1990 cuando se crean las titulaciones Ingeniería Técnica en Informática de Gestión, Ingeniería Técnica de Informática de Sistemas y la Ingeniería Informática. La Resolución de 8 de junio de 2009, de la Secretaría General de Universidades, da publicidad al Acuerdo del Consejo de Universidades, por el que se establecen recomendaciones para la propuesta por las universidades de la memoria de solicitud del título oficial en el ámbito de la Ingeniería Informática. Con esta titulación se pretende formar a profesionales que tengan una formación versátil y transversal, de tal forma que el ingeniero pueda adaptarse fácilmente a distintos entornos de trabajo. De ahí que en la citada resolución aparezcan, entre otras, las competencias genéricas y las específicas que el egresado de esta titulación debe haber adquirido. Según el proyecto Tuning (González \& Wagenaar, 2003):

- Competencias transversales o genéricas: son las que, pese a no estar relacionadas con los conocimientos técnicos propios de la titulación, debe poseer un titulado con ese nivel académico.

- Competencias técnicas o específicas: son las relativas a los conocimientos técnicos propios de la titulación.

En esta investigación, nos centramos en la competencia matemática que es una competencia específica de la titulación. La noción de competencia matemática se ha usado con distintas interpretaciones (Schwartz, 1995; Abrantes, 2001; Schoenfeld, 2007; Rico \& Lupiáñez, 2008; Britta, 2010; Kuntze, 2010). Especial mención merece la definición dada por Niss (2002) según la cual la competencia matemática es la habilidad de entender, juzgar, hacer y usar las matemáticas en una variedad de situaciones y contextos extra matemáticos, en los que éstas juegan o podrían jugar un papel. Destacamos en esta definición la no alusión a contenidos curriculares. Las asignaturas de Matemáticas que de forma obligatoria cursan los Ingenieros Informáticos son (salvo denominaciones que pueden cambiar de una universidad a otra): Álgebra Lineal y Estructuras Matemáticas, Cálculo, Estadística y, Lógica y Métodos Discretos. A partir de la formación matemática que reciben los ingenieros informáticos y que figura, entre otros, en el Plan de Estudios de la Universidad de Granada que está aprobado por la Agencia Nacional de Evaluación de la Calidad y 
Acreditación (ANECA) (Universidad de Granada, 2009), identificamos cinco competencias matemáticas específicas: competencia algebraica, competencia analítica, competencia lógica, competencia estadística y competencia numérica que vienen descritas por las capacidades que aparecen en las tablas III, IV, V, VI y VII, respectivamente. Entendemos por competencia matemática en este estudio como la que viene descrita por las cinco competencias matemáticas específicas anteriores.

En cuanto a los perfiles profesionales del egresado en Ingeniería Informática, usaremos en este trabajo los definidos por Fernández, García, Gaya, Égido, Mata \& Aliane (2006) en el que se tuvieron en cuenta los estudios RENTIC (Requisitos para el Empleo en Nuevas Tecnologías de la Información y las Comunicaciones) (Fernández, 2003) que elabora un análisis de los requisitos incluidos en las ofertas de empleo dirigidas a titulados universitarios de informática publicadas en los principales diarios nacionales así como en otras fuentes de información como bolsas de empleo. Son los siguientes: programador, técnico de sistemas, analista programador, comercial, director de informática, especialista, formador o profesor, analista, técnico de comunicaciones o redes, consultor informático, responsable de proyectos o desarrollo, informático y administrador de bases de datos. Estos perfiles, si bien no coinciden en la denominación de los puestos, encajan con las áreas laborales descritas en Career Space (2001).

\section{Método}

El método empleado para llevar a cabo esta investigación es de tipo cuantitativo (Anderson, 2000), eminentemente descriptivo basado en el uso de una encuesta. El estudio, que es de tipo no experimental o expostfacto, lo hemos llevado a cabo en cuatro fases consecutivas: diseño de la encuesta, validación de la misma por parte de expertos y mediante una prueba piloto, distribución de la encuesta entre los egresados y análisis de los resultados.

\section{Participantes}

La elección de la muestra se basó en lo expuesto por Teichler (2003) que afirma que las encuestas a egresados un año después de acabar la carrera o incluso antes no son fiables y las consideraciones de Raga (2003) que propone como indicador para obtener el porcentaje de titulados que consideran que han conseguido un trabajo satisfactorio, un mínimo de tres años después de terminar sus estudios.

La muestra, que fue incidental, estuvo compuesta por 414 sujetos (46 mujeres y 368 hombres) con una edad comprendida entre los 29 y los 39 años (Edad media $=35,6$; Desviación típica $=3,1$ ) todos ellos egresados con las siguientes titulaciones: Ingeniería Técnica en Informática (15\%), Ingeniería Superior en Informática (64\%) y Doctores en Informática (21\%). El perfil profesional de los egresados que participaron en el estudio, así como su distribución por comunidades autónomas viene dado en la tabla 1. 


\begin{tabular}{|c|c|c|c|c|c|c|c|c|}
\hline 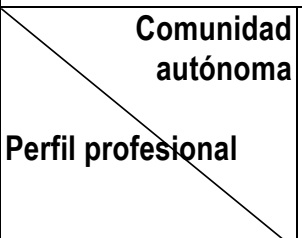 & \begin{tabular}{|l}
$\frac{\pi}{0}$ \\
$\frac{0}{0}$ \\
$\frac{\pi}{0}$ \\
$\frac{0}{2}$
\end{tabular} & 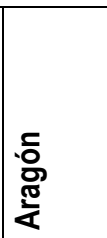 & $\frac{\mathscr{2}}{\frac{\pi}{2}}$ & 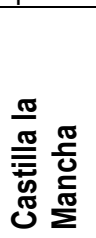 & 总 & 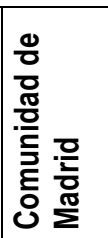 & 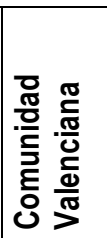 & Total \\
\hline Programador & 12 & 8 & 5 & 5 & 10 & 11 & 7 & 58 \\
\hline Técnico de sistemas & 7 & 5 & 4 & 5 & 8 & 9 & 11 & 49 \\
\hline $\begin{array}{l}\text { Analista } \\
\text { programador }\end{array}$ & 6 & 4 & 3 & 4 & 7 & 10 & 3 & 37 \\
\hline $\begin{array}{l}\text { Director de } \\
\text { informática }\end{array}$ & 8 & 3 & 2 & 3 & 9 & 7 & 3 & 35 \\
\hline Formador o profesor & 5 & 7 & 4 & 6 & 5 & 3 & 4 & 34 \\
\hline Analista & 7 & 2 & 2 & 3 & 5 & 6 & 5 & 30 \\
\hline $\begin{array}{l}\text { Responsable de } \\
\text { proyectos o } \\
\text { desarrollo }\end{array}$ & 2 & 3 & 4 & 5 & 3 & 4 & 7 & 28 \\
\hline $\begin{array}{l}\text { Consultor } \\
\text { informático }\end{array}$ & 4 & 1 & 4 & 6 & 7 & 3 & 2 & 27 \\
\hline Comercial & 3 & 5 & 6 & 3 & 6 & 2 & 1 & 26 \\
\hline Informático & 3 & 4 & 2 & 3 & 4 & 5 & 3 & 24 \\
\hline Especialista & 4 & 3 & 3 & 2 & 5 & 4 & 3 & 24 \\
\hline $\begin{array}{l}\text { Técnico de } \\
\text { comunicaciones o } \\
\text { redes }\end{array}$ & 3 & 2 & 1 & 2 & 5 & 6 & 4 & 23 \\
\hline $\begin{array}{l}\text { Administrador de } \\
\text { bases de datos }\end{array}$ & 3 & 2 & 1 & 2 & 4 & 4 & 3 & 19 \\
\hline Total & 103 & 23 & 29 & 45 & 72 & 91 & 51 & 414 \\
\hline
\end{tabular}

\section{Instrumento}

Para identificar las competencias matemáticas, se utilizó un cuestionario diseñado ad hoc, validado mediante un estudio piloto con 74 sujetos y un juicio de expertos en el que participaron: un ingeniero informático con amplia experiencia docente y profesional, un matemático profesional y un profesor titular de universidad que ha impartido numerosas asignaturas de matemáticas en las titulaciones de Informática. La primera parte del cuestionario contiene 9 ítems con los que se pretende recabar información del egresado relacionada con el sexo, edad, titulación y tipo de trabajo que realiza. A continuación, se formulan 71 preguntas con opciones de respuesta tipo Likert (Hurtado, 2000) y con los siguientes valores: 0 (Nunca), 1 (Muy pocas veces), 2 (Algunas veces) y 3 (Frecuentemente). La distribución de los ítems se hizo en torno a las cinco categorías definidas por las competencias matemáticas específicas y es la que aparece en la tabla 2.

\begin{tabular}{|cc|}
\hline Tabla 2. Número de ítems asignado a cada competencia matemática específica \\
\hline Competencia matemática específica & $\mathbf{N}^{0}{ }^{\circ}$ de ítems \\
\hline Algebraica & 13 \\
Analítica & 7 \\
Lógica & 18 \\
Estadística & 18 \\
Numérica & 15 \\
\hline Total & 71 \\
\hline
\end{tabular}


El alfa de Cronbach para el cuestionario global es de 0,85 y oscila entre 0,71 y 0,79 para las cinco competencias matemáticas específicas. Finalmente, se pidió a los egresados que puntuasen de 1 a 5 las cinco competencias matemáticas específicas antes descritas dándole un 1 a la que consideran que necesitarían más formación académica que la recibida en los estudios universitarios de Ingeniería Informática y un 5 a la que menos.

\section{Procedimiento}

En primer lugar, se diseñó un cuestionario destinado a determinar las competencias matemáticas que un ingeniero informático usa en su trabajo. Para tal efecto, se tomó como referencia el plan de estudios del Grado en Ingeniería Informática impartido en la Universidad de Granada y verificado por ANECA. Una vez validado, se aplicó a la muestra antes descrita a través de una encuesta que cada participante rellenaba online a través de limesurvey.

El proceso de recogida de datos empezó con la obtención de las direcciones de correo electrónico de los participantes en el estudio a través de los Centros de Recogida y Análisis de Información (CRAI) de la Universidad Autónoma de Madrid, Universidad de Granada, Universidad Politécnica de Valencia, Universidad de Zaragoza, Universidad Nacional de Educación a Distancia, Universidad de Valladolid, Universidad de La Coruña, Universidad de Castilla-La Mancha, Universidad de Las Palmas de Gran Canaria y de una búsqueda en la red temática de criptografía y seguridad de la información (CRIPTORED). Seguidamente, se envió a cada egresado un correo electrónico con la presentación de la investigación y con el enlace al documento donde se alojaba el cuestionario. Con el fin de aumentar el número de respuestas se envió un correo electrónico de recordatorio. Para garantizar el anonimato de cada participante se le pidió como primera pregunta del cuestionario que indicase las cuatro últimas cifras de su documento nacional de identidad. Finalmente, se volcaron todos los datos en una base de datos para su posterior análisis.

\section{Resultados}

A continuación, presentamos tabulados los resultados obtenidos en las diferentes competencias matemáticas específicas incluyendo la puntuación promedio y la desviación típica. También introducimos las dos capacidades matemáticas más usadas por los egresados en Ingeniería Informática, las competencias matemáticas demandadas y las competencias matemáticas específicas que usan los diferentes perfiles profesionales. Con el fin de analizar si existen diferencias estadísticamente significativas en el uso de las diferentes competencias matemáticas analizadas en función del sexo, perfil profesional, titulación y comunidad autónoma se aplicó la prueba $\mathrm{U}$ de Mann-Whitney para la variable sexo y la prueba $\mathrm{H}$ de Kruskal-Wallis para el resto, ambas con un nivel de significación de 0,05. El tratamiento estadístico de los datos obtenidos se ha llevado a cabo con el paquete informático SPSS 18.0.

Competencia algebraica: En lo que se refiere a la competencia algebraica, como se puede observar en la tabla III, los ítems más valorados han sido el número $1 \mathrm{y} \mathrm{el} 2$. El rango de puntuaciones oscila entre los 4 puntos del ítem 1 (que es el ítem más valorado de toda la encuesta en su conjunto) y los 1,464 puntos del ítem 11; esto nos da un rango de 2,536 puntos. Para esta competencia no se han encontrado diferencias estadísticamente significativas entre los sujetos de la muestra en función del sexo, perfil profesional y comunidad autónoma. La prueba $\mathrm{H}$ de Kruskal-Wallis nos permite afirmar que sí existen en base a la titulación (véase tabla 8). 


\begin{tabular}{|c|c|c|c|c|}
\hline & Capacidad & $\begin{array}{c}\text { Puntuación } \\
\text { promedio }\end{array}$ & $\begin{array}{l}\text { Desviación } \\
\text { típica }\end{array}$ & $\begin{array}{l}\text { Puntuación promedio de } \\
\text { la competencia }\end{array}$ \\
\hline $\begin{array}{c}\text { İtem } \\
1\end{array}$ & Resolver sistemas de ecuaciones lineales & 4 & 1,414 & \multirow{13}{*}{2,359} \\
\hline $\begin{array}{l}\text { İtem } \\
2\end{array}$ & $\begin{array}{l}\text { Conocer aplicaciones de los sistemas de } \\
\text { ecuaciones lineales }\end{array}$ & 3,035 & 0,922 & \\
\hline $\begin{array}{l}\text { Ítem } \\
3\end{array}$ & Realizar operaciones con matrices & 2,714 & 1,117 & \\
\hline $\begin{array}{c}\text { Ítem } \\
4\end{array}$ & $\begin{array}{c}\text { Interpretar vectores en el espacio } \\
\text { bidimensional y tridimensional de forma } \\
\text { algebraica } \\
\end{array}$ & 2,714 & 1,049 & \\
\hline $\begin{array}{l}\text { Ítem } \\
5\end{array}$ & $\begin{array}{l}\text { Determinar el núcleo y la imagen de una } \\
\text { aplicación lineal }\end{array}$ & 2,678 & 1,156 & \\
\hline $\begin{array}{l}\text { İtem } \\
6\end{array}$ & $\begin{array}{l}\text { Conocer si una matriz es diagonalizable y, en } \\
\text { su caso, diagonalizarla }\end{array}$ & 2,357 & 1,129 & \\
\hline $\begin{array}{l}\text { Ítem } \\
7\end{array}$ & $\begin{array}{c}\text { Conocer aplicaciones de los espacios } \\
\text { vectoriales }\end{array}$ & 1,785 & 0,875 & \\
\hline $\begin{array}{c}\text { Ítem } \\
8\end{array}$ & $\begin{array}{c}\text { Determinar la dimensión de un espacio } \\
\text { vectorial }\end{array}$ & 2,678 & 1,123 & \\
\hline $\begin{array}{c}\text { Ítem } \\
9\end{array}$ & Conocer la aritmética de polinomios & 2,214 & 1,133 & \\
\hline $\begin{array}{c}\text { Ítem } \\
10\end{array}$ & $\begin{array}{l}\text { Saber aplicar la aritmética entera y modular } \\
\text { para resolver problemas }\end{array}$ & 1,892 & 1,030 & \\
\hline $\begin{array}{c}\text { Ítem } \\
11\end{array}$ & Conocer la terminologías básica de conjuntos & 1,464 & 0,576 & \\
\hline $\begin{array}{c}\text { İtem } \\
12\end{array}$ & $\begin{array}{c}\text { Saber aplicar la teoría de grafos para resolver } \\
\text { problemas }\end{array}$ & 1,535 & 0,637 & \\
\hline $\begin{array}{c}\text { Ítem } \\
13\end{array}$ & $\begin{array}{c}\text { Utilizar software simbólico para resolver } \\
\text { problemas de naturaleza algebraica }\end{array}$ & 1,607 & 0,785 & \\
\hline
\end{tabular}

Competencia analítica: Con respecto a la competencia analítica (tabla 4), los ítems con mayores puntuaciones han sido el número 14 y el 15 . El rango de puntuaciones oscila entre los 2,714 puntos del ítem 14 y los 1,678 puntos del ítem 19; esto nos da un rango de 1,036 puntos. Como se puede observar en la tabla 8 , no existen diferencias estadísticamente significativas entre la utilización de esta competencia y el sexo, perfil profesional, titulación y comunidad autónoma, debido a que los niveles de significación son mayores que 0,05 .

\begin{tabular}{|c|c|c|c|c|}
\hline & Capacidad & $\begin{array}{l}\text { Puntuación } \\
\text { promedio }\end{array}$ & $\begin{array}{l}\text { Desviación } \\
\text { típica }\end{array}$ & $\begin{array}{l}\text { Puntuación promedio de } \\
\text { la competencia }\end{array}$ \\
\hline $\begin{array}{l}\text { Ítem } \\
14\end{array}$ & $\begin{array}{l}\text { Conocer las propiedades de las funciones y } \\
\text { sus inversas }\end{array}$ & 2,714 & 1,013 & \multirow{7}{*}{2,091} \\
\hline $\begin{array}{l}\text { Ítem } \\
15\end{array}$ & $\begin{array}{c}\text { Usar analíticamente las funciones } \\
\text { elementales }\end{array}$ & 2,464 & 0,838 & \\
\hline $\begin{array}{l}\text { Ítem } \\
16\end{array}$ & Representar gráficamente funciones & 1,964 & 0,792 & \\
\hline $\begin{array}{l}\text { İtem } \\
17\end{array}$ & Resolver problemas de optimización & 2 & 0,816 & \\
\hline $\begin{array}{l}\text { Ítem } \\
18\end{array}$ & Utilizar algún software de cálculo simbólico & 2,107 & 0,994 & \\
\hline $\begin{array}{l}\text { Ítem } \\
19\end{array}$ & $\begin{array}{l}\text { Entender y aplicar los conceptos de derivada } \\
\text { e integral (indefinida y definida) }\end{array}$ & 1,678 & 0,818 & \\
\hline $\begin{array}{l}\text { Ítem } \\
20\end{array}$ & $\begin{array}{l}\text { Entender y aplicar los conceptos de límite y } \\
\text { continuidad de funciones }\end{array}$ & 1,714 & 0,896 & \\
\hline
\end{tabular}


Competencia lógica: En la tabla 5 se observa que los ítems más valorados para la competencia lógica han sido el número 36 y el 37 . El rango de puntuaciones oscila entre los 2,607 puntos del ítem 36 y los 1,25 puntos del ítem 35; esto nos da un rango de 1,357 puntos. La prueba de $\mathrm{H}$ de Kruskal-Wallis nos muestra que existen diferencias estadísticamente significativas entre el uso de esta competencia y el perfil profesional. Por el contrario, no se han encontrado diferencias estadísticamente significativas entre el uso de la competencia y el sexo, titulación y comunidad autónoma (véase tabla 8).

\begin{tabular}{|c|c|c|c|c|}
\hline & Capacidad & $\begin{array}{l}\text { Puntuación } \\
\text { promedio }\end{array}$ & $\begin{array}{l}\text { Desviación } \\
\text { típica }\end{array}$ & $\begin{array}{l}\text { Puntuación promedio de } \\
\text { la competencia }\end{array}$ \\
\hline $\begin{array}{c}\text { İtem } \\
21\end{array}$ & Conocer las álgebras de Boole finitas & 2,178 & 1,188 & \multirow{18}{*}{1,852} \\
\hline $\begin{array}{l}\text { Ítem } \\
22\end{array}$ & Aplicar el concepto de función booleana & 1,714 & 0,854 & \\
\hline $\begin{array}{l}\text { Ítem } \\
23 \\
\end{array}$ & $\begin{array}{c}\text { Conocer las formas normales de expresiones } \\
\text { booleanas }\end{array}$ & 2,178 & 1,248 & \\
\hline $\begin{array}{l}\text { İtem } \\
24\end{array}$ & $\begin{array}{c}\text { Saber aplicar el algoritmo de Quine- } \\
\text { McCluskey }\end{array}$ & 2,285 & 1,150 & \\
\hline $\begin{array}{l}\text { Ítem } \\
25\end{array}$ & $\begin{array}{c}\text { Conocer la semántica del lenguaje } \\
\text { proposicional }\end{array}$ & 1,428 & 0,690 & \\
\hline $\begin{array}{l}\text { Ítem } \\
26\end{array}$ & Saber aplicar la implicación semántica & 1,75 & 0,967 & \\
\hline $\begin{array}{l}\text { Ítem } \\
27\end{array}$ & Conocer el teorema de la deducción & 1,892 & 1,165 & \\
\hline $\begin{array}{l}\text { İtem } \\
28\end{array}$ & Saber aplicar la forma normal conjuntiva & 1,821 & 0,983 & \\
\hline $\begin{array}{l}\text { Ítem } \\
29\end{array}$ & Aplicar el algoritmo de Davis-Putnam & 2,035 & 0,961 & \\
\hline $\begin{array}{c}\text { Ítem } \\
30\end{array}$ & Conocer los conjuntos de Horn & 1,428 & 0,790 & \\
\hline $\begin{array}{c}\text { Ítem } \\
31\end{array}$ & $\begin{array}{c}\text { Conocer la semántica de la lógica de primer } \\
\text { orden }\end{array}$ & 2,071 & 1,152 & \\
\hline $\begin{array}{l}\text { Ítem } \\
32\end{array}$ & Aplicar el algoritmo de unificación & 1,428 & 0,835 & \\
\hline $\begin{array}{l}\text { İtem } \\
33\end{array}$ & $\begin{array}{l}\text { Representar ideas usando el lenguaje de la } \\
\text { lógica }\end{array}$ & 1,357 & 0,678 & \\
\hline $\begin{array}{l}\text { Ítem } \\
34\end{array}$ & $\begin{array}{c}\text { Resolver problemas lógicos usando } \\
\text { herramientas de la lógica matemática }\end{array}$ & 1,321 & 0,611 & \\
\hline $\begin{array}{l}\text { Ítem } \\
35\end{array}$ & Utilizar circuitos lógicos combinacionales & 1,25 & 0,585 & \\
\hline $\begin{array}{l}\text { Ítem } \\
36\end{array}$ & Aplicar el principio de inducción matemática & 2,607 & 1,100 & \\
\hline $\begin{array}{l}\text { Ítem } \\
37\end{array}$ & $\begin{array}{l}\text { Utilizar software para resolver problemas de } \\
\text { naturaleza lógica }\end{array}$ & 2,535 & 0,999 & \\
\hline $\begin{array}{l}\text { İtem } \\
38\end{array}$ & $\begin{array}{l}\text { Conocer aplicaciones de la lógica } \\
\text { proposicional y de predicados }\end{array}$ & 2,071 & 1,119 & \\
\hline
\end{tabular}

Competencia estadística: Con respecto a la competencia estadística (tabla 6), los ítems con puntuaciones más elevadas han sido el número 40 y el 39 . El rango de puntuaciones oscila entre los 2,571 puntos del ítem 40 y los 1,714 puntos del ítem 51; esto nos da un rango de 0,857 puntos que es el menor de todas las competencias estudiadas. 
Analizando los resultados de la tabla VIII, podemos afirmar que no existen diferencias estadísticamente significativas entre el uso de esta competencia y el sexo, titulación, comunidad autónoma y perfil profesional.

\begin{tabular}{|c|c|c|c|c|}
\hline & Capacidad & $\begin{array}{l}\text { Puntuación } \\
\text { promedio }\end{array}$ & $\begin{array}{l}\text { Desviación } \\
\text { típica }\end{array}$ & $\begin{array}{l}\text { Puntuación } \\
\text { promedio de la } \\
\text { competencia }\end{array}$ \\
\hline $\begin{array}{c}\text { Ítem } \\
39\end{array}$ & $\begin{array}{l}\text { Distinguir entre un estudio observacional y un } \\
\text { experimento }\end{array}$ & 2,428 & 0,997 & \multirow{18}{*}{2,063} \\
\hline $\begin{array}{c}\text { Ítem } \\
40\end{array}$ & Analizar la representatividad de una muestra & 2,571 & 0,920 & \\
\hline $\begin{array}{c}\text { Ítem } \\
41\end{array}$ & $\begin{array}{c}\text { Decidir qué gráfico es el más adecuado para cada } \\
\text { variable estadística }\end{array}$ & 2,142 & 0,890 & \\
\hline $\begin{array}{c}\text { Ítem } \\
42\end{array}$ & Saber interpretar cualquier gráfico estadístico & 2,107 & 1,065 & \\
\hline $\begin{array}{c}\text { Ítem } \\
43\end{array}$ & Saber clasificar una variable estadística & 2,107 & 0,994 & \\
\hline $\begin{array}{c}\text { Ítem } \\
44\end{array}$ & Identificar errores en gráficos estadísticos & 2,178 & 0,944 & \\
\hline $\begin{array}{c}\text { Ítem } \\
45\end{array}$ & Saber interpretar los parámetros de centralización & 1,857 & 0,755 & \\
\hline $\begin{array}{c}\text { Ítem } \\
46\end{array}$ & $\begin{array}{c}\text { Conocer la interpretación de los parámetros de } \\
\text { dispersión }\end{array}$ & 2,035 & 0,881 & \\
\hline $\begin{array}{c}\text { Ítem } \\
47\end{array}$ & Saber interpretar las medidas de curtosis & 2,25 & 0,751 & \\
\hline $\begin{array}{c}\text { Ítem } \\
48\end{array}$ & $\begin{array}{l}\text { Identificar en un espacio muestral los sucesos } \\
\text { elementales, complementarios, compatibles e } \\
\text { independientes }\end{array}$ & 1,964 & 0,838 & \\
\hline $\begin{array}{c}\text { Ítem } \\
49\end{array}$ & $\begin{array}{c}\text { Aplicar la regla de la multiplicación para sucesos } \\
\text { independientes, la regla de la suma para la unión de } \\
\text { sucesos y la regla del complementario }\end{array}$ & 2,142 & 0,890 & \\
\hline $\begin{array}{c}\text { Ítem } \\
50\end{array}$ & $\begin{array}{c}\text { Encontrar el z-valor en una Distribución Normal para } \\
\text { determinar percentiles }\end{array}$ & 1,75 & 0,887 & \\
\hline $\begin{array}{c}\text { Ítem } \\
51\end{array}$ & $\begin{array}{l}\text { Usar la tabla de la Distribución Normal Standard para } \\
\text { calcular probabilidades }\end{array}$ & 1,714 & 0,809 & \\
\hline $\begin{array}{c}\text { Ítem } \\
52\end{array}$ & Conocer el contraste de hipótesis estadísticas & 1,928 & 1,051 & \\
\hline $\begin{array}{c}\text { Ítem } \\
53\end{array}$ & $\begin{array}{l}\text { Saber aplicar las técnicas de regresión y correlación } \\
\text { lineal a situaciones reales }\end{array}$ & 1,750 & 1,004 & \\
\hline $\begin{array}{c}\text { Ítem } \\
54\end{array}$ & $\begin{array}{l}\text { Conocer las técnicas estadísticas adecuadas para } \\
\text { resolver problemas de la Investigación Operativa }\end{array}$ & 2,142 & 1,145 & \\
\hline $\begin{array}{c}\text { Ítem } \\
55\end{array}$ & $\begin{array}{c}\text { Saber resolver cuestiones relacionadas con la } \\
\text { Inferencia Estadística }\end{array}$ & 2,000 & 0,942 & \\
\hline $\begin{array}{c}\text { Ítem } \\
56\end{array}$ & Utilizar software estadístico para resolver problemas & 2,071 & 1,086 & \\
\hline
\end{tabular}

Competencia numérica: Los ítems más valorados para la competencia numérica, según figura en la tabla 7 , han sido el número 62 y el 57 . El rango de puntuaciones oscila entre los 2,821 puntos del ítem 62 y los 1,035 puntos del ítem 69; esto nos da un rango de 1,786 puntos.

Como se puede observar en la tabla VIII, al ser los niveles de significación superiores a 0,05 , no existen diferencias estadísticamente significativas entre el uso de esta competencia y el sexo, titulación, perfil profesional y comunidad autónoma. 


\begin{tabular}{|c|c|c|c|c|}
\hline & Capacidad & $\begin{array}{l}\text { Puntuación } \\
\text { promedio }\end{array}$ & $\begin{array}{c}\text { Desviación } \\
\text { típica }\end{array}$ & $\begin{array}{c}\text { Puntuación promedio } \\
\text { de la competencia }\end{array}$ \\
\hline $\begin{array}{c}\text { İtem } \\
57\end{array}$ & $\begin{array}{l}\text { Conocer métodos numéricos para resolver } \\
\text { ecuaciones algebraicas y trascendentes }\end{array}$ & 2,392 & 0,994 & \multirow{15}{*}{1,840} \\
\hline $\begin{array}{c}\text { Ítem } \\
58\end{array}$ & $\begin{array}{c}\text { Saber aplicar métodos numéricos apropiados para } \\
\text { aproximar una función }\end{array}$ & 1,607 & 0,831 & \\
\hline $\begin{array}{c}\text { Ítem } \\
59\end{array}$ & $\begin{array}{c}\text { Conocer métodos numéricos adecuados para } \\
\text { resolver una ecuación diferencial }\end{array}$ & 1,714 & 0,854 & \\
\hline $\begin{array}{c}\text { Ítem } \\
60\end{array}$ & $\begin{array}{c}\text { Aplicar métodos numéricos apropiados para } \\
\text { evaluar la derivada de una función }\end{array}$ & 1,571 & 0,878 & \\
\hline $\begin{array}{c}\text { Ítem } \\
61\end{array}$ & $\begin{array}{l}\text { Poner en práctica métodos numéricos adecuados } \\
\text { para resolver un sistema de ecuaciones lineales }\end{array}$ & 1,571 & 0,790 & \\
\hline $\begin{array}{c}\text { Ítem } \\
62\end{array}$ & $\begin{array}{c}\text { Conocer métodos numéricos para resolver } \\
\text { ecuaciones no lineales }\end{array}$ & 2,821 & 1,090 & \\
\hline $\begin{array}{c}\text { Ítem } \\
63\end{array}$ & $\begin{array}{l}\text { Utilizar un procedimiento de análisis de errores } \\
\text { para un método numérico dado }\end{array}$ & 2,107 & 0,956 & \\
\hline İtem & $\begin{array}{c}\text { Analizar y evaluar la precisión de los métodos } \\
\text { numéricos utilizados }\end{array}$ & 1,964 & 0,961 & \\
\hline $\begin{array}{l}\text { İtem } \\
65\end{array}$ & $\begin{array}{c}\text { Calcular una integral definida usando un método } \\
\text { numérico apropiado }\end{array}$ & 1,535 & 0,744 & \\
\hline $\begin{array}{l}\text { Ítem } \\
66\end{array}$ & $\begin{array}{l}\text { Saber implementar métodos numéricos en el } \\
\text { software conveniente }\end{array}$ & 2,071 & 0,857 & \\
\hline $\begin{array}{l}\text { İtem } \\
67\end{array}$ & $\begin{array}{c}\text { Aplicar métodos numéricos para obtener } \\
\text { soluciones aproximadas a problemas de la } \\
\text { Ingeniería Informática }\end{array}$ & 1,714 & 0,762 & \\
\hline $\begin{array}{c}\text { Ítem } \\
68\end{array}$ & Conocer las técnicas de interpolación & 1,714 & 0,809 & \\
\hline $\begin{array}{l}\text { İtem } \\
69\end{array}$ & $\begin{array}{c}\text { Aplicar técnicas numéricas para el ajuste de } \\
\text { curvas }\end{array}$ & 1,035 & 0,961 & \\
\hline $\begin{array}{c}\text { Ítem } \\
70 \\
\end{array}$ & $\begin{array}{c}\text { Conocer técnicas numéricas de resolución de } \\
\text { ecuaciones diferenciales ordinarias }\end{array}$ & 1,535 & 0,744 & \\
\hline $\begin{array}{c}\text { Ítem } \\
71\end{array}$ & $\begin{array}{l}\text { Analizar la velocidad de convergencia de un } \\
\text { método numérico iterativo }\end{array}$ & 2,250 & 1,109 & \\
\hline
\end{tabular}

Tabla 8. Nivel de significación de contrastes no paramétricos $\mathrm{U}$ de Mann-Whitney y $\mathrm{H}$ de Kruskall-Wallis entre las competencias matemáticas y variables clasificatorias

\begin{tabular}{|cccccc|}
\hline & Algebraica & Analítica & Lógica & Estadística & Numérica \\
\hline Perfil profesional &, 621 &, 532 &, 008 &, 436 &, 006 \\
Titulación &, 009 &, 176 &, 298 &, 201 &, 569 \\
Comunidad autónoma &, 765 &, 421 &, 089 &, 431 &, 407 \\
Sexo &, 322 &, 598 &, 190 &, 256 &, 753 \\
\hline
\end{tabular}

Competencias matemáticas específicas que utilizan los distintos perfiles profesionales: A continuación, presentamos una tabla en la que aparecen por filas los perfiles profesionales de los ingenieros informáticos y por columnas las competencias matemáticas específicas. Indicamos para cada perfil profesional de mayor a menor intensidad $(1>2>3>4>5)$ las competencias más usadas. 


\begin{tabular}{|c|c|c|c|c|c|c|}
\hline \multirow{14}{*}{$\begin{array}{c}\text { Perfil } \\
\text { profesional }\end{array}$} & \multirow[b]{2}{*}{ Programador } & \multicolumn{5}{|c|}{ Competencias matemáticas específicas } \\
\hline & & 2 & 3 & 5 & 4 & 1 \\
\hline & Técnico de sistemas & 1 & 3 & 4 & 5 & 2 \\
\hline & Analista programador & 3 & 2 & 5 & 4 & 1 \\
\hline & Director de informática & 2 & 5 & 1 & 3 & 4 \\
\hline & Formador o profesor & 2 & 3 & 5 & 4 & 1 \\
\hline & Analista & 2 & 4 & 5 & 1 & 3 \\
\hline & $\begin{array}{c}\text { Responsable de proyectos o } \\
\text { desarrollo }\end{array}$ & 2 & 5 & 1 & 4 & 3 \\
\hline & Consultor informático & 2 & 5 & 4 & 1 & 3 \\
\hline & Comercial & 4 & 3 & 1 & 2 & 5 \\
\hline & Informático & 2 & 3 & 5 & 4 & 1 \\
\hline & Especialista & 2 & 3 & 5 & 4 & 1 \\
\hline & Técnico de comunicaciones o redes & 2 & 3 & 4 & 5 & 1 \\
\hline & Administrador de bases de datos & 2 & 3 & 4 & 5 & 1 \\
\hline
\end{tabular}

Observamos que la competencia estadística es la más usada por directores de informática, responsables de proyectos o desarrollo y comerciales. La competencia numérica es la que más utilizan en su trabajo los analistas y consultores informáticos. Los programadores, analistas programadores, formadores o profesores, informáticos, especialistas, técnicos de comunicaciones o redes y administradores de bases de datos usan con más frecuencia la competencia lógica en su labor profesional. Finalmente, la competencia algebraica es la más usada por técnicos de sistemas.

Capacidades matemáticas más usadas por los informáticos egresados: Un aspecto que puede resultar de interés es conocer las dos capacidades matemáticas más utilizadas por los ingenieros informáticos en el ejercicio de su profesión. En la tabla $X$ figuran tabuladas las dos capacidades más usadas por cada uno de los distintos perfiles profesionales con las respectivas puntuaciones promedio $y$ desviaciones típicas:

\begin{tabular}{|c|c|c|c|}
\hline \multicolumn{4}{|c|}{ Tabla 10. Capacidades más usadas por los ingenieros informáticos egresados } \\
\hline Tipo de trabajo & Capacidades más utilizadas & $\begin{array}{l}\text { Puntuación } \\
\text { promedio }\end{array}$ & $\begin{array}{l}\text { Desviación } \\
\text { típica }\end{array}$ \\
\hline \multirow[t]{2}{*}{ Programador } & $\begin{array}{l}\text { 1. Conocer la semántica de la lógica de primer } \\
\text { orden }\end{array}$ & 2,752 & 0,887 \\
\hline & $\begin{array}{l}\text { 2. Utilizar software para resolver problemas de } \\
\text { naturaleza lógica }\end{array}$ & 2,690 & 0,919 \\
\hline \multirow[t]{2}{*}{ Técnico de sistemas } & $\begin{array}{l}\text { 1. Conocer aplicaciones de los sistemas de } \\
\text { ecuaciones lineales }\end{array}$ & 2,772 & 0,658 \\
\hline & $\begin{array}{l}\text { 2. Saber aplicar la aritmética entera y modular } \\
\text { para resolver problemas }\end{array}$ & 2,711 & 0,989 \\
\hline \multirow[t]{2}{*}{ Analista programador } & 1. Conocer las álgebras de Boole finitas & 2,601 & 1,010 \\
\hline & $\begin{array}{l}\text { 2. Utilizar software para resolver problemas de } \\
\text { naturaleza lógica }\end{array}$ & 2,566 & 0,901 \\
\hline \multirow[t]{2}{*}{ Comercial } & 1. Analizar la representatividad de una muestra & 2,882 & 0,781 \\
\hline & 2. Saber interpretar cualquier gráfico estadístico & 2,769 & 0,941 \\
\hline \multirow[t]{2}{*}{ Director de informática } & 1. Analizar la representatividad de una muestra & 2,709 & 0,795 \\
\hline & $\begin{array}{l}\text { 2. Saber aplicar las técnicas de regresión y } \\
\text { correlación lineal a situaciones reales }\end{array}$ & 2,602 & 0,816 \\
\hline Especialista & \begin{tabular}{|lll} 
1. Resolver problemas lógicos usando \\
herramientas de la lógica matemática
\end{tabular} & 2,409 & 1,078 \\
\hline
\end{tabular}




\begin{tabular}{|c|c|c|c|}
\hline & $\begin{array}{l}2 \text { Utilizar software para resolver problemas de } \\
\text { naturaleza lógica }\end{array}$ & 2,370 & 0,791 \\
\hline \multirow[t]{2}{*}{ Formador/profesor } & 1. Conocer las álgebras de Boole finitas & 2,488 & 0,898 \\
\hline & $\begin{array}{l}\text { 2. Utilizar software estadístico para resolver } \\
\text { problemas }\end{array}$ & 2,412 & 0,901 \\
\hline \multirow[t]{2}{*}{ Analista } & $\begin{array}{l}\text { 1. Entender y aplicar los conceptos de derivada } \\
\text { e integral (indefinida y definida) }\end{array}$ & 2,512 & 0,689 \\
\hline & 2. Saber interpretar cualquier gráfico estadístico & 2,479 & 0,678 \\
\hline \multirow[t]{2}{*}{$\begin{array}{c}\text { Técnico de } \\
\text { comunicaciones/redes }\end{array}$} & \begin{tabular}{lllll|} 
1. Conocer aplicaciones de la lógica \\
proposicional y de predicados
\end{tabular} & 2,309 & 0,751 \\
\hline & $\begin{array}{l}\text { 2. Saber aplicar la teoría de grafos para resolver } \\
\text { problemas }\end{array}$ & 2,251 & 0,992 \\
\hline \multirow[t]{2}{*}{ Consultor informático } & 1. Utilizar algún software de cálculo simbólico & 2,690 & 0,841 \\
\hline & 2. Analizar la representatividad de una muestra & 2,551 & 1,102 \\
\hline \multirow{2}{*}{$\begin{array}{c}\text { Responsable de } \\
\text { proyectos/desarrollo }\end{array}$} & 1. Saber interpretar cualquier gráfico estadístico & 2,751 & 0,765 \\
\hline & $\begin{array}{l}\text { 2. Saber resolver cuestiones relacionadas con la } \\
\text { Inferencia Estadística }\end{array}$ & 2,554 & 0,891 \\
\hline \multirow[t]{2}{*}{ Informático } & $\begin{array}{|llll|}\text { 1. Conocer aplicaciones de la lógica } \\
\text { proposicional y de predicados }\end{array}$ & 2,887 & 0,794 \\
\hline & $\begin{array}{l}\text { 2. Analizar y evaluar la precisión de los métodos } \\
\text { numéricos utilizados }\end{array}$ & 2,697 & 0,598 \\
\hline \multirow[t]{2}{*}{$\begin{array}{l}\text { Administrador de base de } \\
\text { datos }\end{array}$} & $\begin{array}{l}\text { 1. Saber aplicar la aritmética entera y modular } \\
\text { para resolver problemas }\end{array}$ & 2,798 & 0,581 \\
\hline & $\begin{array}{l}\text { 2. Saber aplicar la teoría de grafos para resolver } \\
\text { problemas }\end{array}$ & 2,690 & 0,708 \\
\hline
\end{tabular}

\section{Competencias matemáticas demandadas por los ingenieros informáticos egresados}

Un aspecto interesante es conocer las competencias matemáticas sobre las que los egresados demandan más formación académica que la recibida en sus estudios universitarios para desempeñar de manera eficiente su labor profesional. En la tabla $X$ indicamos de mayor a menor intensidad $(1>2>3>4>5)$ las competencias más demandadas para cada perfil profesional.

\begin{tabular}{|c|c|c|c|c|c|c|}
\hline & & \multicolumn{5}{|c|}{ Competencias matemáticas específicas } \\
\hline & & \multicolumn{5}{|c|}{ Algebraica Analítica Estadística Numérica Lógica } \\
\hline \multirow{13}{*}{$\begin{array}{c}\text { Perfil } \\
\text { profesional }\end{array}$} & Programador & 3 & 2 & 5 & 4 & 1 \\
\hline & Técnico de sistemas & 3 & 2 & 5 & 1 & 4 \\
\hline & Analista programador & 4 & 3 & 2 & 1 & 5 \\
\hline & Director de informática & 4 & 3 & 1 & 2 & 5 \\
\hline & Formador o profesor & 4 & 3 & 2 & 1 & 5 \\
\hline & Analista & 3 & 4 & 2 & 1 & 5 \\
\hline & Responsable de proyectos o desarrollo & 4 & 3 & 2 & 1 & 5 \\
\hline & Consultor informático & 4 & 3 & 1 & 2 & 5 \\
\hline & Comercial & 5 & 3 & 1 & 4 & 2 \\
\hline & Informático & 3 & 2 & 5 & 1 & 4 \\
\hline & Especialista & 4 & 2 & 5 & 1 & 3 \\
\hline & Técnico de comunicaciones o redes & 3 & 4 & 2 & 1 & 5 \\
\hline & Administrador de bases de datos & 4 & 3 & 1 & 2 & 5 \\
\hline
\end{tabular}

Con respecto a las necesidades de formación matemática que manifiestan los sujetos de la muestra, observamos que la competencia estadística es la más demandada por directores de informática, consultores informáticos y comerciales. La competencia numérica es la que más demandan en su trabajo los técnicos de sistemas, analistas programadores, formadores o profesores, analistas, responsables de proyectos 0 
desarrollo, informáticos, especialistas y técnicos de comunicaciones o redes. Los programadores necesitarían más formación matemática para desarrollar su competencia lógica.

\section{Discusión}

Uno de los objetivos del presente estudio ha consistido en determinar las competencias matemáticas específicas más usadas por los ingenieros informáticos egresados en su ejercicio profesional. Como segundo objetivo, se han mostrado las competencias matemáticas específicas (algebraica, analítica, lógica, estadística y numérica) sobre las que estos egresados necesitarían más formación que la recibida en sus estudios universitarios para ejercer su labor profesional de manera eficiente.

La puntuación promedio en cuanto al uso de la competencia matemática (la hemos calculado como la media aritmética de las puntuaciones promedio de las cinco competencias matemáticas específicas estudiadas) por parte de los ingenieros informáticos en su labor profesional es de 2,054 que se corresponde con un valor de 'Algunas veces' lo que nos revela la importancia que tiene el desarrollo de esta competencia en su formación académica. Este resultado va en la línea de los obtenidos por Pérez Iglesias (2012) y Vintere \& Zeidmane (2014).

Particularizando en las competencias matemáticas específicas, los resultados obtenidos son muy satisfactorios siendo la competencia algebraica la más usada con un puntuación promedio (calculada como la media aritmética de las puntuaciones promedios de las capacidades que describen cada competencia) de 2,359 puntos (equivalente a un uso de la competencia 'Algunas veces'), seguida por la competencia analítica, y la competencia numérica la menos usada con una puntuación promedio de 1,840 puntos (equivalente a un uso de la competencia muy cercano a 'Algunas veces'). Esto nos lleva a sugerir que se potencie la formación de los ingenieros informáticos tanto en Álgebra como en Cálculo, siguiendo las indicaciones de Cardella (2008).

La capacidad más usada por los egresados, con una puntuación promedio de 4 , es "Resolver sistemas de ecuaciones lineales", que forma parte de la competencia algebraica. La menos usada, con un promedio de 1,035 es "Aplicar técnicas numéricas para el ajuste de curvas", que describe la competencia numérica.

La dispersión de las respuestas respecto a la puntuación promedio de las capacidades medidas en este estudio oscila entre 0,576 puntos (ítem 11) y 1,414 puntos (ítem 1).

La competencia matemática específica para la que demandan más formación los egresados para realizar de manera eficiente su labor profesional es la competencia numérica, que no coincide con la competencia matemática más utilizada.

En esta investigación hemos usado los perfiles profesionales definidos por Fernández et ál. (2006) que son los siguientes: programador, técnico de sistemas, analista programador, comercial, director de informática, especialista, formador o profesor, analista, técnico de comunicaciones o redes, consultor informático, responsable de proyectos o desarrollo, informático y administrador de bases de datos. Centrándonos en estos perfiles, hemos observado que los comerciales, directores de informática y responsables de proyectos o desarrollo usan con mayor frecuencia en su labor profesional la competencia estadística mientras que los administradores de bases de datos, técnicos de comunicaciones o redes, especialistas, informáticos, profesores o formadores, analistas programadores y los programadores usan la competencia 
numérica. Por otro lado, los técnicos de sistemas hacen uso con mayor asiduidad de la competencia lógica. La competencia matemática más usada por los ingenieros informáticos coincide con la más demandada en el caso de los programadores (competencia lógica), analistas (competencia numérica) y directores de informática y comerciales (competencia matemática). Coincidimos con Walesh (2006) cuando afirma que las matemáticas y, por tanto, el desarrollo de su competencia matemática es muy importante en la formación de los ingenieros ya que es una herramienta que les permite resolver problemas extra matemáticos.

Sería interesante que el sistema universitario español llevase a cabo estudios periódicos para determinar las competencias matemáticas que usan los egresados en el ejercicio de su profesión y las tuviese en cuenta en la elaboración de los planes de estudio.

El haber contado con una muestra formada por egresados de todas las comunidades y ciudades autónomas le habría dado al estudio una mayor representatividad.

Una línea de trabajo futura podría ser analizar las competencias matemáticas usadas por ingenieros informáticos de otros países en el ejercicio de su profesión y realizar un análisis comparativo. Este estudio se podría extender a titulaciones en las que las competencias que exige el mercado se tengan que comparar con respecto a las competencias que los estudiantes desarrollan en sus estudios universitarios; por ejemplo: el Grado en Administración y Dirección de Empresas y el Grado en Ingeniería de Tecnologías de Telecomunicación.

\section{Referencias}

Abrantes, P. (2001). Mathematical competence for all: Options, implications and obstacles. Educational Studies in Mathematics, 47, $125-143$.

Anderson, G. (2000). Fundamentals of Education Research. London: Basingstoke, Falmer. ANECA (2004). Libro Blanco. Título de Grado en Ingeniería Informática. Recuperado de

Ávila, M. \& Aguirre, C. (2005). El seguimiento de los egresados como indicador de la calidad docente. REIFOP, 8 (3).

Britta, C. (2010). Heureka - ich hab's. Stationsarbeit zur Problemlösekompetenz. Mathematik 5 bis 10, 13, $26-29$.

Carbonell, L. (2002). Un análisis de los estudios universitarios de Informática a través de sus egresados. El caso de Alicante (1984-2001). Tesis doctoral. Universidad de Alicante. Recuperado de http://hdl.handle.net/10045/3355

Cardella, M. (2008). Which mathematics should we teach engineering students? An empirically grounded case for a broad notion of mathematical thinking. Teaching Mathematics and its Applications 27(3), pp. 150-159.

Career Space (2001). Perfiles de capacidades profesionales genéricas de TIC. Capacidades profesionales futuras para el mundo del mañana. Centro Europeo para el Desarrollo de la Formación Profesional: Bruselas. Recuperado de https://www.fi.upm.es/docs/estudios/grado/901_CareerSpace-Profiles.pdf

Fernández, L. (2003). Requisitos para el empleo en Nuevas Tecnologías de la Información: el estudio RENTIC. Revista Novótica, 161.

Fernández, L., García, M. J., Gaya, M. C., Égido, V., Mata, M. \& Aliane, N. (2006). Análisis de empleabilidad y perfiles de egresado en las titulaciones de informática en España. XII Jornadas sobre la Enseñanza Universitaria de la Informática, Deusto, 12-14 julio.

Freire, M. J., Teijeiro, M. M., \& Pais, C. (2013). La adecuación entre las competencias adquiridas por los graduados y las requeridas por los empresarios. Revista de Educación, 362, 13-41.

González, J. \& Wagenaar, R. (Eds.) (2003). Tuning Educational Structures in Europe. Informe Final. Fase 1. Bilbao, España: Universidad de Deusto. 
Hurtado, J. (2000). Metodología de la investigación holística. Caracas: Editorial Sypal.

Jaramillo, A., Giraldo, A. \& Ortiz, J. S. (2006). Estudios sobre egresados. La experiencia de la Universidad EAFIT. Revista Universidad EAFIT, 42 (141), 111-124

kuntze, S. (2010). Zur Beschreibung von Kompetenzen des mathematischen Modellierens konkretisiert an inhaltlichen Leitideen: Eine Diskussion von Kompetenzmodellen als Grundlage für Förderkonzepte zum Modellieren im Mathematikunterricht. Mathematikunterricht (Seelze), 56 (4), 4-19.

López Ruiz, J. (2010). Un giro copernicano en la enseñanza no universitaria: formación por competencias. Revista de Educación, 356, $279-301$.

Nguyen, D. (1998). The essential skills and attributes of an engineer: a comparative study of academics, industry personnel and engineering students. Global Journal of Engineering Education, 2 (1).

Niss, M. (2002). Mathematical competencies and the learning of mathematics: The Danish kom project. Recuperado de http://www.math.chalmers.se/Math/Grundutb/CTH/mve375/1213/docs/KOMkompetenser.pdf

OCDE (2005). The definition and selection of key competencies. Executive summary. París: OCDE.

OCDE (2008). Tertiary Education for the knowledge Society. París: OCDE.

Pérez Iglesias, J. L. (2012). Competencias que demandan los egresados en Ingeniería en el ámbito de la Informática y los egresados en Ingeniería en el ámbito industrial. Tesis doctoral. Universidad de Salamanca. Recuperado de http://hdl.handle.net/10366/121211

Radcliffe, D. (2006). Global challenges facing Engineering Education: opportunities for innovation. $35^{\text {th }}$ International IGIP Symposium in cooperation with IEEE/ASEE/SEFI. Tallín, 18 septiembre.

Raga, J.T. (2003). Presentación. En J. Vidal (Coord.) Métodos de análisis de la inserción laboral de los universitarios:Seminario. León: Secretariado de Publicaciones y Medios Audiovisuales. Recuperado de http://sid.usal.es/idocs/F8/FDO7238/estudio.pdf

Rico, L. \& Lupiáñez, J. L. (2008). Competencias matemáticas desde una perspectiva curricular. Madrid, España: Alianza Editorial.

Schoenfeld, A. (2007). Assessing Mathematical Proficiency. University Press, Cambridge.

Schwartz, R. (1995). Is Mathematical Competence Innate? Philosophy of Science, 62 (2), 227-240.

Tascón, A., Álvarez, R., Couto, A., Gutiérrez, P. \& Aguado, P. (2013). Estudio de inserción laboral y competencias en ingenieros agrónomos titulados por la Universidad de León. Actas del VII Congreso Ibérico de Agroingeniería y Ciencias Hortícolas. Recuperado de http://sechaging-madrid2013.org/geystiona/adjs/comunicaciones/272/C02360002.pdf

Teichler, U. (2003). Aspectos metodológicos de las encuestas a graduados universitarios. Centro para la Investigación en educación Superior y Trabajo. En J. Vidal (Coord.) Métodos de análisis de la inserción laboral de los universitarios: Seminario. León: Secretariado de Publicaciones y Medios Audiovisuales. Recuperado de http://sid.usal.es/idocs/F8/FDO7238/estudio.pdf

Universidad de Granada (2009). Memoria verificada del Grado en Ingeniería Informática de la Universidad de Granada. Recuperado de http://secretariageneral.ugr.es/bougr/pages/desarrollo/2009/271109/ingenieriainformatica271109/!

Urrego-Giraldo, G. y Giraldo, G. L. (2009). Incorporación de las competencias al modelo de construcción de currículos de ingeniería basados en problemas: el caso de la ingeniería de sistemas. Revista Avances en Sistemas e Informática, 6(3), 33-43.

Vidal, J. (Coord.) (2003). Métodos de análisis de la inserción de los universitarios. Salamanca: Consejo de Coordinación Universitaria.

Vintere, A. \& Zeidmane, A. (2014). Mathematics studies at university: effect on the professional competence. International Scientifical Conference, Jelgava, 23-24 mayo.

Walesh, S. G. (2006). Body of Knowledge for Civil Engineers: Essential for Success in the International Arena. 35 th International IGIP Symposium in cooperation with IEEE / ASEE / SEFI, Tallín, 18-09-2006. 